\title{
A Statistical Approach for Post-Processing Residential Building Energy Simulation Output
}

\author{
S. Valovcin ${ }^{\mathrm{a}, \mathrm{b}}$, A. S. Hering ${ }^{\mathrm{a}, *}$, B. Polly ${ }^{\mathrm{b}}$, M. Heaney $^{\mathrm{b}}$ \\ ${ }^{a}$ Department of Applied Mathematics and Statistics, Colorado School of Mines, Golden, \\ CO, United States \\ ${ }^{b}$ Buildings and Thermal Systems Center, National Renewable Energy Laboratory, \\ Golden, CO, United States
}

\begin{abstract}
Residential building energy simulation (RBES) software plays an important role in evaluating the energy consumption and efficiency potential of homes. These physics-based models are commonly used to assess the energy performance of homes and to predict benefits of making energy-saving improvements to homes a priori. However, software may produce biased estimates of energy consumption for a variety of reasons, including: errors in the measurement and observation of building characteristics; differences in the assumed versus actual occupant behavior; and errors in the physical models and algorithms used in the software. In order to evaluate and improve the accuracy of RBES software, the National Renewable Energy Laboratory (NREL) has assembled a set of approximately 1,250 U.S. homes for which measured energy consumption and audit-collected household energy characteristics are available. Algorithms have also been developed that
\end{abstract}

\footnotetext{
*Corresponding author

Email addresses: svalovcin@gmail.com (S. Valovcin), ahering@mines.edu (A. S. Hering), ben.polly@nrel.gov (B. Polly), michael.heaney@nrel.gov (M. Heaney)
} 
automatically translate the data from each home into RBES input files so that model predictions of annual electricity and natural gas consumption can be compared to measured values. To assess and improve upon the accuracy of these predictions, we first cluster the homes using weighted, independent linear combinations of these variables and then build multiple linear regressions within clusters of similar homes to model the difference between measured and predicted energy consumption based on the recorded features of the homes. The statistical post-processing techniques that we develop for RBES models have the following benefits: (1) they can identify variables and algorithms that may be causing inaccuracies in the RBES process, and (2) they can be used to adjust and improve the RBES predictions. Keywords: Clustering, Cross-validation, Model calibration, Principal component analysis, Residential building energy simulation

1. Introduction

In 2010, the residential sector accounted for $23 \%$ of the United States' 3 total energy consumption, which is $4.4 \%$ of the world's energy consumption 4 [1]. Efforts to understand and model this consumption are critical to reducing 5 it. Residential Building Energy Simulation (RBES) tools typically model the energy consumption (e.g., electricity and natural gas) of a home at hourly or sub-hourly time steps using a physics-based approach. ${ }^{1}$ Both federally and privately funded organizations have developed such tools for use in home

\footnotetext{
${ }^{1}$ Some RBES tools may use larger time-steps or seasonal approaches for modeling; so long as the tool is able to predict annual consumption, the statistical techniques applied in this study are relevant.
} 
energy assessments that help energy efficiency practitioners and homeowners evaluate the economic benefit of investing in home improvements designed to reduce energy consumption.

One particular quantity of interest predicted by an RBES program is the pre-retrofit annual energy consumption of a home. Then, the annual energy consumption of a home under proposed retrofits can be projected, and the estimated energy savings can be compared with the cost of the retrofit. However, physics-based models can provide biased predictions of the annual energy consumption of the home. The top panels of Figure 1 show a sample of homes for which RBES predictions of annual energy consumption are plotted against the weather-normalized measured annual energy. Natural gas predictions align better with the line of perfect agreement than the electricity predictions do, but the RBES model still tends to overpredict consumption for this sample of homes. Both electricity and natural gas consumption can be highly driven by occupant behavior, but the errors in electricity predictions are thought to be even more variable due to the fact that a large fraction of electric end use is almost entirely driven by occupant preferences and choices (e.g., miscellaneous electric loads, appliances, and lighting). However, in this study, standard occupant behavior is used in the software, and the building characteristics data were generally collected for the purpose of an "asset rating," which means specific occupant behavior was not considered. Thus, standard occupant behavior is assumed to provide a fair comparison of the homes' energy efficiency features.

An approach for adjusting predictions to more closely match the actual electric energy consumption in the home would be useful, as shown in the 
bottom panels of Figure 1, which will be discussed in more detail in Section 4 .

Deviations between observed and predicted energy consumption may occur for any of the following reasons: (1) measurement error in data collection; (2) error in utility data normalization; (3) deviations in occupant behavior; (4) coding errors in translation scripts; or (5) deficiencies in the RBES model $[2]$.

This research focuses on the last two types of errors. First, in order for a home to be successfully simulated by an RBES tool, a specific set of simulation inputs describing the home is required. Translation scripts automatically map information in a database for a given home to create a simulation input file, as shown in Figure 2. Some inputs are computed based on characteristics observed or measured in the home. For example, duct leakage percentages are calculated when the results of a duct pressurization test that is performed during the audit are available. Assumptions, simplifications, and coding errors in the translation scripts can contribute to errors in the RBES predictions. The RBES program may also face certain limitations, like the inability to model homes with multiple hot water heaters or multiple duct systems. In such cases, assumptions and simplifications are necessary to make predictions.

Secondly, there may be errors and inaccuracies in the RBES program itself. Default assumptions made within the program, such as occupant use profiles, may not be accurate for the particular homes being analyzed. Inherent simplifications, such as the use of an isothermal, single-zone model for the conditioned space, could introduce significant error into the model predictions. Modeling algorithms, such as ground-coupling models for foun- 


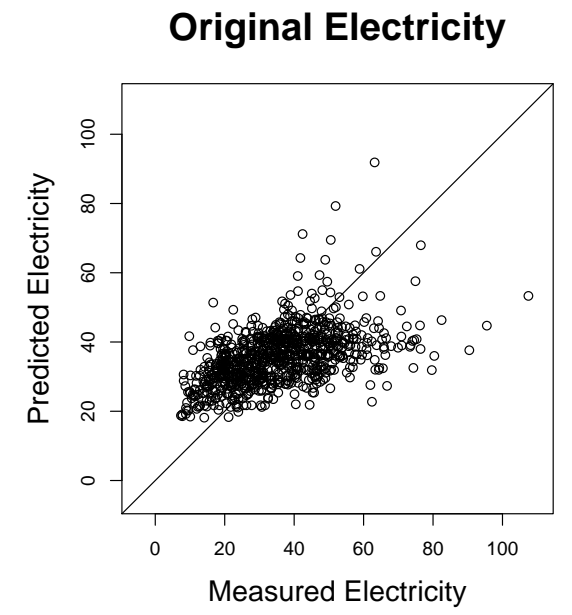

Adjusted Electricity

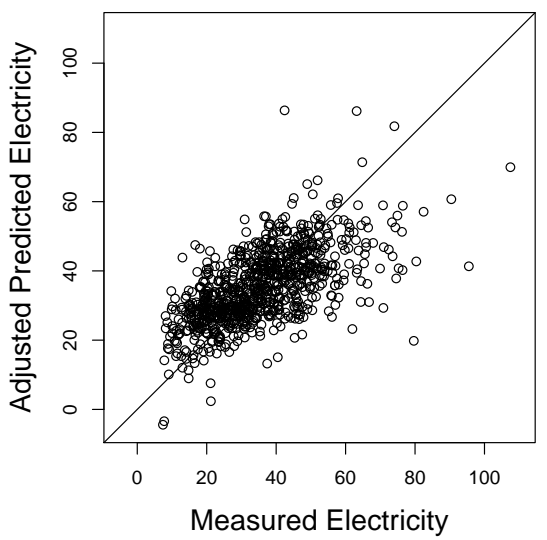

\section{Original Natural Gas}

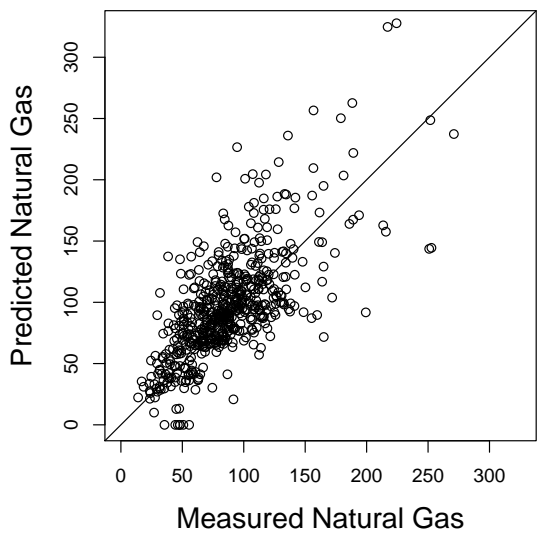

Adjusted Natural Gas

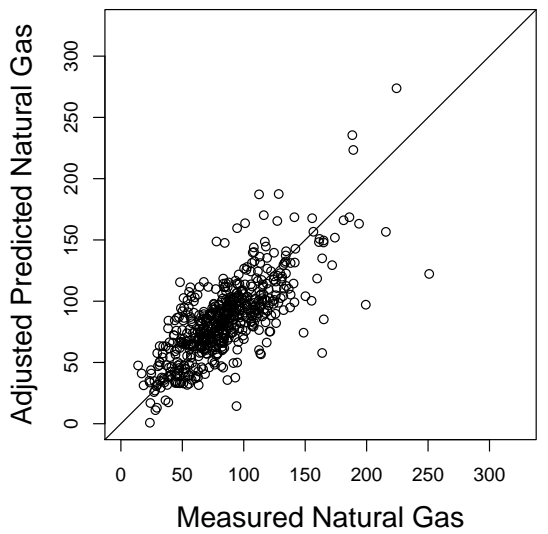

Figure 1: Top Row: Plot of RBES predictions of electricity (left) and natural gas (right) against weather-normalized measured annual consumption of each type of energy in MMBtu. Bottom Row: Plot of adjusted RBES predictions versus weather-normalized measured annual electricity (left) and natural gas (right) consumption for the combined clusters with a reference line of perfect agreement. 


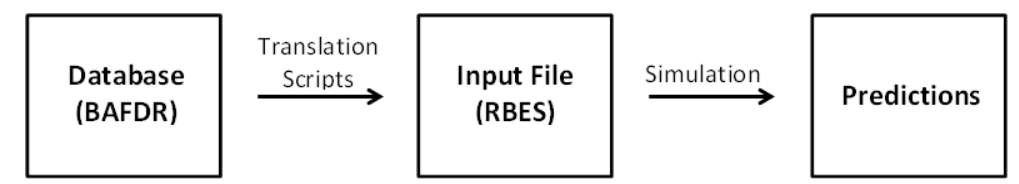

Figure 2: Outline of general RBES simulation process. The BAFDR is NREL's Building America Field Data Repository that contains data on the homes used in this study.

dation heat transfer, may not accurately model the energy performance of a particular component of the home. There may even be coding errors in the RBES program itself, which can contain hundreds-of-thousands of lines of computer code.

Several prior studies have sought to assess various RBES models by comparing predictions to measured energy, but most only assess accuracy on a relatively small sample of homes [2-5]. EnergyPro was found to, on average, overestimate the combined electricity and natural gas savings expected from retrofits by nearly a half in a sample of 51 homes [5]. However, the Home Energy Scoring Tool (HEST) only slightly underestimated electricity $(n=859)$ and underestimated natural gas consumption in $60 \%$ of the 500 homes that were examined [6]. In the HEST study, multiple linear regression (MLR) models for the difference between predicted and measured annual energy consumption were built, and significant variables in these models were noted as inputs that could be investigated to identify potential model improvements. However, the HEST model does not require many inputs, most of them are categorical, and the majority of the inputs used in the accuracy assessment were HEST inputs. For this study, we include more variables, many of which are numerical instead of categorical, and we also consider variables in the statistical analysis that are not inputs to the simulation tool 
but perhaps could improve the accuracy of the tool if they were. All of these prior studies stopped short of post-processing the RBES predictions to improve them, but one analysis of the SEEM model does use building characteristics and energy consumption data to adjust SEEM predictions of total heating energy to obtain improved estimates of electric heating energy [7]. In that work, the adjustment is also made using a multiple linear regression model with a sample size of 629 homes, but only one model is built for all homes in the study, and only a small set of variables is used.

Post-processing physics-based models began with numerical weather prediction models in the early 1970's [8,9] and has been shown to improve forecasts made by these models by correcting biases and adapting forecasts to local conditions. Since then, variations of Model Output Statistics (MOS) have been applied to many atmospheric variables, including temperature, ozone, wind speeds, and precipitation (e.g., [10-14]). The general principle is that the physical model captures the nonlinearities of the large-scale system while the statistical post-processing calibrates the model output to local conditions. Using a large suite of homes with measured energy consumption that have been simulated using an RBES program, we apply such an approach to the RBES predictions and can correct the predictions for bias and reduce variability in the predictions. As a by-product, we can also identify variables that may be miscoded in the translation scripts or that may be related to inaccuracies in the RBES model.

Using physical characteristics recorded for each home, variables calculated with the translation scripts, and RBES model input variables, MLR models are built for the difference between the measured and predicted an- 
nual electricity and natural gas consumption. Homes are first put into similar groups using a statistical clustering technique, and MLR models built within each cluster are shown to have better predictive accuracy than models built using all homes. Clustering offers a way to construct similar groups for observations with large dimensionality, and at least two prior building energy studies have also demonstrated improved results when clustering is applied first $[15,16]$. MLR models for the groups of homes allow us to pinpoint areas of improvement in the RBES process that are specific to the homes in each group.

The remainder of this paper is organized as follows: Section 2 describes the RBES model, translation scripts, and the homes used in this analysis. Section 3 describes the methodology, including the cluster analysis on principal components, MLR models, and leave-one-out cross-validation. The results are illustrated in Section 4, and Section 5 concludes with a discussion of the practical impact of this work and future questions of interest.

\section{Experimental Data}

The methodology and results in this work are based on the energy consumption and recorded features of a set of approximately 1,250 homes in the National Renewable Energy Laboratory's (NREL's) Building America Field Data Repository (BAFDR). The electricity and natural gas consumption of a subset of these homes is predicted using NREL's Building Energy Optimization Software (BEopt ${ }^{\mathrm{TM}}$ / EnergyPlus) and is the particular RBES model that we use to obtain energy predictions. However, the methodology described herein could apply to any RBES tool. Descriptions of the BAFDR 
homes, their recorded features, and the BEopt simulation tool are given in this section. This section also includes a summary of the translation process, which takes the original information recorded for each home and converts it to BEopt inputs.

\subsection{BAFDR Data}

The data used in this study is a subset of homes from NREL's Building America Field Data Repository (BAFDR), which is a database containing energy audit information and measured energy use for approximately 1,250 homes (at the time this study was conducted) that have been collected from 8 different sources, which are listed in Table 1 of the Appendix. The variables recorded for each home include information on actual energy consumption, ${ }^{2}$ location, weather, and household characteristics. The household characteristics cover a broad range of variables that are used to describe features of each home related to its geometry, wall construction, attic and roof construction, foundation, windows, infiltration, ducts, ventilation, appliances, space conditioning, and water heating.

\footnotetext{
${ }^{2}$ Unless the measured energy consumption data were originally received in a weathernormalized format, utility billing data were weather normalized using the ASHRAE Inverse Modeling Toolkit (IMT) software program. Three parameter change-point heating models were used with natural gas billing data, and both three and five parameter heating and cooling change-point models were used with electricity billing data. For cases with insufficient goodness-of-fit $\left(R^{2}<0.7\right)$, the model coefficients were not used, and an annual average energy use was calculated from the utility billing data. See "ASHRAE Research Project 1050-RP: Development of a Toolkit for Calculating Linear, Change-Point Linear and Multiple-Linear Inverse Building Energy Analysis Models" [17], which includes background information about IMT and the models.
} 


\subsection{RBES Process}

The BEopt (Building Energy Optimization) software has been developed by NREL in support of the U. S. Department of Energy Building America program. BEopt evaluates residential building designs and identifies cost-optimal efficiency packages at various levels of whole-house energy savings along the path to zero net energy, which is when a home consumes as much energy as it produces annually. Both new construction and existing home retrofits can be analyzed through evaluation of single building designs, parametric sweeps, and cost-based optimizations. BEopt provides detailed simulation-based analysis using specific house characteristics, such as size, architecture, occupancy, vintage, location, and utility rates using existing, established simulation engines (currently DOE-2.2 or EnergyPlus). For this study, BEopt 2.0 Beta is used with the EnergyPlus v 7.1 simulation engine (DOE 2013). This version of BEopt is not capable of and is not intended to be used for simulating the energy consumption of certain homes in the BAFDR, such as complex multi-family buildings and homes with multiple duct systems.

At the time this study was completed, translation algorithms and associated computer scripts were being developed at NREL that automatically translate BAFDR data to create BEopt extensible markup language (XML) input files for each home. A development version of these translation scripts was used in this study for the secondary purpose of testing and improving the translation algorithms. Automated simulation approaches are gaining traction as data transfer standards like Home Performance XML (HPXML) are becoming more widely used. Large datasets that are stored in a standard file 
format, such as the BAFDR, will allow industry software developers to apply empirical testing and software corrections using the approaches presented in this study.

The development version of the translation algorithms were written to handle the most common features of homes in the BAFDR, such as woodframe walls, but were not capable of processing uncommon technologies like SIP walls or geothermal heat pumps. The homes used in this project are the 997 single-family, detached homes in the BAFDR that were successfully translated and whose electricity consumption could be simulated in BEopt. Note, a subset of 709 of these homes had non-zero measured natural gas usage and were also simulated in BEopt. While BEopt's user interface allows homes to be described using predefined options, the household characteristics collected from homes in the BAFDR are generally not described using these options. Instead, custom BEopt options were developed for these homes based on the information given in the BAFDR, such as the duct and infiltration variables. Furthermore, there is no information on occupants in the BAFDR database, so the homes were simulated assuming "typical occupant behavior," which refers to the standardized occupancy defined in the Building America House Simulation Protocols [18].

Given that there are several steps to the simulation process, the variables used in this study come from multiple points in this process, as depicted in Figure 2. Specifically, the 105 household characteristics we use come from (i) the BAFDR data, (ii) intermediate steps in the translation scripts, and (iii) the BEopt input files. While approximately $60 \%$ of the variables are straight from the BEopt input file, most of the geometry related variables come from 
the BAFDR (20\%), and all of the variables related to U-values are from the translation scripts (20\%). Variables are included in the analysis if they directly represent or are derived from collected information about the home. For a complete list of variables used, their types, definitions, and summary statistics, see Appendix A.

Studies that use many variables describing features of the same home often find that redundancies in the variables can cause numerical instabilities [7]. For example, both the BAFDR and BEopt have different representations of floor area. Called multicollinearity, this phenomenon occurs when one variable is a linear combination of the remaining variables in the set. In this project, the presence of multicollinearity presents the problem of overstating similarities and differences between homes in the cluster analysis, and it can have a large impact on fitting multiple linear regression (MLR) models, as it causes the estimates of the model coefficients to be unstable with large standard errors. Multicollinearity can be detected using variance inflation factors (VIFs) that describe the percentage of variability in one particular variable that can be described as a linear function of the remaining variables. The rule of thumb is that variables with VIFs larger than 10 are removed [19].

Given that many of the variables present in the BAFDR, intermediate set, and BEopt inputs are related, the VIFs are used to identify redundant variables before subsequent analysis is performed. The subset of variables is chosen by removing variables one at a time based on large VIFs and expert opinion. This process is iterated until all but one variable, Heating Degree Days, have a VIF less than 10. The VIF for Heating Degree Days is just 
greater than 10, but since it is considered to be important from a building energy standpoint, it is retained. Thus, a subset of 49 of the original 105 variables that are nearly independent is used in this analysis.

The top panels of Figure 1 show the RBES predicted versus measured annual energy consumption for the electricity and natural gas consumption of the homes, respectively. The observations that fall below the line of perfect agreement are homes for which the simulation process underpredicts energy consumption, and the observations above the line are homes that are overpredicted by the simulation process. We define $\Delta$ Electricity to be the difference between a set of RBES model predictions and measured electricity (RBES Predicted - Measured), and $\Delta$ Natural Gas is defined similarly. Table 1 gives the mean and standard deviation of these differences and of the measured electricity and natural gas. From this, we see that across all homes, electricity is overpredicted by $0.8 \%$ on average, and natural gas is overpredicted by $10.1 \%$ on average. While across all homes, the electricity predictions do not appear strongly biased, within each group (which will be described in Section 4.1), larger discrepancies appear.

\section{Methodology}

In this section, we first describe the statistical clustering technique that is applied based on the set of 49 explanatory variables to divide the homes into similar groups. The principal components of the 49 variables are first computed, then two clustering methods are applied, and multiple methods for choosing the number of clusters are tested. Then, the creation of multiple linear regression (MLR) models for each of the $\Delta$ energy responses is briefly 
Table 1: Mean and standard deviation of the measured annual energy and deviations between RBES predicted and measured annual energy given in MMBtu.

\begin{tabular}{|c|c|c|c|c|c|}
\hline \multirow[b]{2}{*}{ Grouping } & \multicolumn{2}{|c|}{$\begin{array}{l}\text { Measured } \\
\text { Electricity }\end{array}$} & \multicolumn{3}{|c|}{$\Delta$ Electricity } \\
\hline & Mean & $\mathrm{SD}$ & Mean & $\mathrm{SD}$ & Bias \\
\hline All Homes & 35.41 & 14.85 & 0.29 & 12.77 & $0.8 \%$ \\
\hline Warm Cluster & 40.37 & 15.00 & -1.96 & 13.50 & $-4.9 \%$ \\
\hline \multirow[t]{2}{*}{ Cold Cluster } & 30.63 & 14.85 & 2.46 & 11.63 & $8.0 \%$ \\
\hline & \multicolumn{2}{|c|}{$\begin{array}{c}\text { Measured } \\
\text { Natural Gas }\end{array}$} & \multicolumn{3}{|c|}{$\Delta$ Natural Gas } \\
\hline Grouping & Mean & $\mathrm{SD}$ & Mean & $\mathrm{SD}$ & Bias \\
\hline All Homes & 87.00 & 37.25 & 9.56 & 32.04 & $10.1 \%$ \\
\hline Warm Cluster & 62.31 & 31.34 & 3.62 & 30.70 & $4.2 \%$ \\
\hline Cold Cluster & 95.02 & 35.48 & 11.48 & 32.27 & $18.4 \%$ \\
\hline
\end{tabular}

described along with a cross-validation technique for adjusting the RBES predictions based on the fitted MLR models.

\subsection{Clustering Analysis}

Three main steps in performing the clustering analysis are described here. First, we obtain principal components (PCs) of the 49 explanatory variables. Even though the explanatory variables were checked for strong multicollinearity, some dependence among them remains, and the PCs produced are independent linear combinations of the original variables. Next, we apply two clustering algorithms to find similar groupings of homes. Since clustering algorithms may be sensitive to assumptions or initial conditions, two such 
algorithms are tested to check for the robustness of the identified clusters. Finally, we apply three methods to select the number of clusters. The number of clusters chosen may also vary depending on which criterion is minimized, so using three different criteria will aid in validating the final choice.

\subsubsection{Principal Component Analysis}

Principal Component Analysis (PCA) is a multivariate statistical technique that transforms the original set of $p$ variables into a new set of $p$ variables through an eigendecomposition of the covariance or correlation matrix. Given that the variables in the BAFDR data set have very different units, we use the correlation matrix for the PCA. Let $\mathbf{X}^{\prime}=\left[X_{1}, X_{2}, \ldots, X_{p}\right]$ be a set of $p$ random variables. To compute the correlation between two quantitative variables, Pearson's product-moment correlation coefficient is used, which is given as

$$
\rho_{i j}=\frac{\operatorname{cov}\left(X_{i}, X_{j}\right)}{\sigma_{X_{i}} \sigma_{X_{j}}},
$$

where $\operatorname{cov}\left(X_{i}, X_{j}\right)$ is the covariance of $X_{i}$ and $X_{j}$, and $\sigma_{X_{i}}$ and $\sigma_{X_{j}}$ are the standard deviations of $X_{i}$ and $X_{j}$, respectively. Pearson's correlation coefficient is chosen since it is mathematically equivalent to the phi coefficient, which we use to measure the association between two binary variables [20], and the point-biserial correlation coefficient, which we use to measure association between continuous and binary variables [21].

The correlation matrix, $\boldsymbol{\rho}$, of $\mathbf{X}$ has an eigenvalue-eigenvector decomposition with pairs $\left\{\left(\lambda_{1}, \mathbf{e}_{\mathbf{1}}\right),\left(\lambda_{2}, \mathbf{e}_{\mathbf{2}}\right), \ldots,\left(\lambda_{p}, \mathbf{e}_{\mathbf{p}}\right)\right\}$, where $\lambda_{1} \geq \lambda_{2} \geq \ldots \geq \lambda_{p} \geq$ 0 . Then, the $k^{t h} \mathrm{PC}$ is given by

$$
Y_{k}=\mathbf{e}_{k}^{\prime} \mathbf{X}=e_{k 1} X_{1}+e_{k 2} X_{2}+\ldots+e_{k p} X_{p}
$$


for $k=1,2, \ldots, p$. The variance of $Y_{k}$ is $\lambda_{k}$, and when the eigenvalues, $\left\{\lambda_{1}, \lambda_{2}, \ldots, \lambda_{p}\right\}$, are distinct, the PCs are independent and have maximum variance with decreasing magnitude [22]. For example, the first PC, $Y_{1}$, has the largest variance of $\lambda_{1}$, followed by the second PC with the second largest variance of $\lambda_{2}$, and so on. PCA is commonly used to reduce the dimensionality of a dataset by representing a large set of $p$ variables with a smaller set of PCs by truncating those PCs that do not describe a large percentage of the variability in $\mathbf{X}$.

\subsubsection{Clustering Methods}

Cluster analysis is an exploratory technique used to find "natural" groupings in multidimensional data. The groupings are determined based on a measure of dissimilarity computed between each pair of homes, and no assumptions are made about the number of groups or the group structure [22]. We cluster observations using the PCs since the PCs are independent, linear combinations of the original variables that can be weighted by their corresponding eigenvalue. In other words, PCs that describe more of the variability in $\mathbf{X}$ (i.e., those with a higher value of $\lambda_{k}$ ) should be allowed to influence the dissimilarity of two homes more than a PC that explains very little of the variability in $\mathbf{X}$. Given that the PCs are numeric, Euclidean distances can be used to measure dissimilarity between pairs of homes, and the distances can be weighted by the corresponding importance of each PC. The weighted Euclidean distance between two $p \times 1$ vectors of principal components, $\mathbf{y}_{k}$ and $\mathbf{y}_{j}$, is given by

$$
d_{i j}=\sqrt{\sum_{m=1}^{p} w_{m}\left(y_{k m}-y_{j m}\right)^{2}}
$$


where $w_{m}$ is equal to $\frac{\lambda_{m}}{\sum_{m=1}^{p} \lambda_{m}}$.

For each pair of homes in a set of $n$ homes, $d_{i j}$ is computed, and these distances are organized into an $n \times n$ matrix, and many methods for clustering observations based on such a matrix have been developed. We apply Ward's method of hierarchical clustering that is based on minimizing the within cluster variance, calculated using the error sum of squares (ESS) [23]. As with all agglomerative clustering methods, each cluster is initially made up of one observation. At each step, the ESS is computed for the union of every possible pair of clusters, and the pair with the smallest increase in ESS is joined. To check the robustness of the clusters that are formed, we also apply the non-hierarchical technique of fuzzy clustering [24]. For a preselected number of clusters, fuzzy clustering assigns each observation a probability of belonging to each cluster, known as the "strength of membership," by minimizing an objective function based on the dissimilarities.

\subsubsection{Choosing the Number of Clusters}

The ideal number of clusters is not known, and many methods exist for choosing the number of clusters. We consider the following three common approaches: the Xie-Beni Index [25], C Index [26], and Dunn Index [27]. Each is based on some comparison of a criteria (such as squared deviations between pairs of homes) between those observations within a cluster and observations assigned to different clusters. Each criteria is computed for different numbers of clusters for each clustering technique, and the number of clusters for which the minimum (or maximum) occurs is chosen. If all three criteria choose a similar optimal number of clusters, then we will have greater confidence in the choice of number of clusters. 


\subsection{MLR Models}

Multiple linear regression analysis is a technique used to assess the relationship of a response variable to a set of explanatory variables. The goal of regression analysis is to fit an empirical model that explains the effects of the explanatory variables on the response [19]. Using the original 49 explanatory variables, we will create models for the following two responses:

- $\Delta$ electricity (RBES predicted - measured) and

- $\Delta$ natural gas (RBES predicted - measured)

both for all homes simultaneously and for the subsets of homes within each identified cluster. We use the original variables in the models instead of the PCs because they are more interpretable, and the presence of particular explanatory variables may point to errors in the translation scripts or areas of potential improvement in the RBES tool. These regression models will indicate how much of the remaining variability in the $\Delta$ variables can be explained by characteristics of the home and will point to those variables that are significant in explaining the difference between predicted and measured energy consumption.

To build each model, all explanatory variables are initially included, and variables are removed one at a time, beginning with the variable with the largest individual $p$-value for significance. This backward elimination process is continued until all remaining variables are significant with a $p$-value less than 0.05 , or all variables have been removed from the model. Once this model is built, the fundamental assumptions of an MLR model are validated. Techniques for checking these assumptions are described in any introductory 
MLR text, such as $[19,28]$, and since we did not find any violations of the assumptions in any of our fitted models, we do not mention this issue again. After assessing and comparing the fitted MLR models, we also assess their predictive accuracy and describe how the MLR predicted values can be used to improve the RBES predictions.

\subsubsection{Leave-one-out Cross-Validation}

A common technique used to assess the predictive capability of a method is leave-one-out cross-validation (CV) in which one observation is removed from the modeling process, and a model fit with the remaining observations is used to predict the removed one. While this method can be computationally expensive, it is commonly used when the sample size is small [29]. Given our relatively small sample size and the even smaller sample size within each cluster, a leave-one-out CV technique is employed to compare the predictive capabilities of the overall model based on all observations versus the models built within each cluster.

First, we assume that the removal of one observation will not significantly impact the correlation matrix, and therefore, the PCs will not change. If the PCs are unchanged, then the dissimilarities based on the eigenvalues and their associated PCs will remain the same. Finally, we also assume that removing one home will not affect the number of clusters chosen. The basic steps for this application of leave-one-out CV are:

1. Remove observation $i$, where $i=1,2, \ldots, n$.

2. Recluster the remaining $n-1$ homes using Ward's method into the predetermined number of clusters. (Fuzzy clustering produced similar results.) 
3. Assign observation $i$ to cluster $C$ by calculating the distance between its variable values and the mean of each cluster and choosing the cluster with the smallest distance.

4. Select the variables (via backward selection) and estimate the coefficients for an MLR model for one of the two responses using all of the observations in $C$ except for observation $i$.

5. Calculate the predicted value, $\hat{y}_{-i}$, for obs $i$ based on this model.

6. Repeat steps 1 through 5 for each observation.

Then, the CV mean-squared error (MSE) is calculated as follows:

$$
C V_{M S E}=\frac{1}{n} \sum_{i=1}^{n}\left(y_{i}-\hat{y}_{-i}\right)^{2},
$$

where $y_{i}$ is the observed value of the response for observation $i$.

\subsubsection{Adjusting RBES Model Output}

Finally, the left-out predictions, $\hat{y}_{-i}$, described in the previous section are independent predictions of how much the RBES tool annual energy predictions will be in error relative to the weather-normalized measured energy use. We claim that these are "independent" predictions since the observation is not used in determining the clusters, is only assigned to a cluster after the clusters have been formed, and is not used to estimate the parameters in the MLR model. Of course, we do assume that the withheld home is similar enough to the remaining homes that it will fit well within one of the clusters and will not require extrapolation of the MLR model to predict its $\Delta$ response. Then, we can obtain an adjusted RBES prediction, $\mathrm{RBES}_{a d j, i}$, by subtracting $\hat{y}_{-i}$ from the original RBES prediction, $\operatorname{RBES}_{i}$. If the RBES prediction error is expected to be positive, subtracting the positive error from 
the RBES prediction should reduce the prediction error (and vice versa for a prediction error that is expected to be negative). We can compute these adjusted prediction errors, $\Delta_{a d j, i}$, as follows:

$$
\begin{aligned}
\Delta_{a d j, i} & =R B E S_{a d j, i}-M_{i} \\
& =\left(R B E S_{i}-\hat{y}_{-i}\right)-M_{i},
\end{aligned}
$$

where $M_{i}$ is the measured annual energy consumption for home $i$. We hope to see a reduction in the bias and in the variability of the $\Delta_{a d j, i}$ values over the $\Delta$ 's in Table 1.

\section{Results and Discussion}

Two clusters are indicated in the cluster analysis, and these are described first. Then, the MLR models that are built within each cluster and for all of the observations for each response are reported and discussed. We demonstrate that splitting the observations into two clusters produces better results than treating all of the observations simultaneously, and more insight is gained into improvements that may be made to the RBES process. Finally, we show that the MLR leave-one-out predictions can adjust the RBES model output to remove the bias and reduce variability.

\subsection{Clustering Analysis}

Figure 3 shows the dendrogram for Ward's method of hierarchical clustering using weighted Euclidean distances based on the PCs. A dendrogram is a visual display used in hierarchical clustering, where the branches represent the clusters, and the height is the value of the metric used to measure 


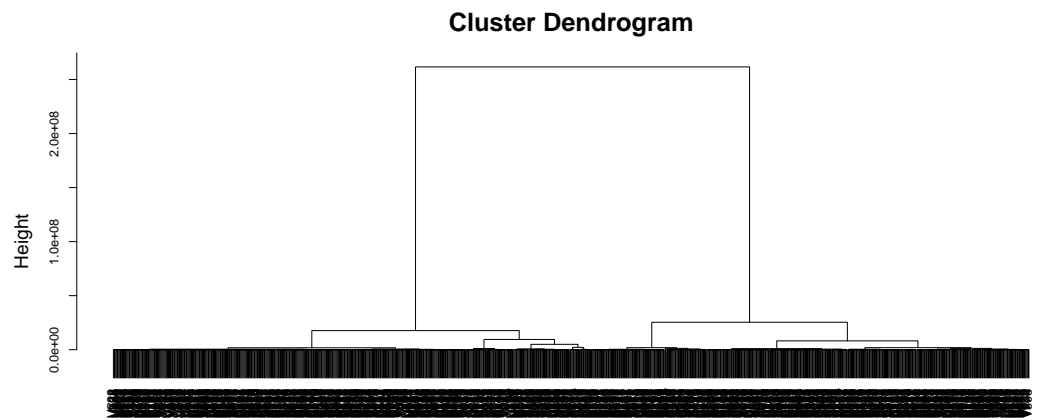

Figure 3: Dendrogram for Ward's method using weighted Euclidean distances of the principal components.

"distance between clusters" when two clusters are joined. These clusters are well-formed; in other words, no homes remain in their own cluster until the last steps, and while the homes along the bottom of the plot are indistinguishable, there is a clear separation between one and two clusters. The clusters identified by the fuzzy method are also well defined.

All three criteria for choosing the number of clusters indicate that two clusters are optimal for both Ward's method and fuzzy clustering. For Ward's method, there are 520 homes in the first cluster and 477 homes in the second. Similarly, there are 507 homes in the first cluster and 490 homes in the second for fuzzy clustering. The clusters are very stable regardless of whether Ward or fuzzy clustering is used since only thirteen homes change clusters. Going forward, we use the clusters formed by Ward's method for the regression analysis, since Ward's method is less computationally expensive.

We label the two clusters as follows: even though the coastal region of Oregon is considered to be a marine climate, to simplify discussion the homes in the first cluster are labeled as "Warm," and the homes in the second cluster 
are labeled as "Cold." The location of the homes at the city level are shown in Figure 4. Cities with red circles indicate that the homes are located in the first Warm cluster, while cities with blue circles indicate that the homes are located in the second Cold cluster. Moreover, the more homes located in a particular city, the darker the color. As shown in Table 1, electricity consumption is underpredicted by $4.9 \%$ on average for Warm cluster homes and overpredicted by $8 \%$ on average for Cold cluster homes. Natural gas consumption is overpredicted by $4.2 \%$ on average for Warm cluster homes and $18.4 \%$ on average for Cold cluster homes.

Clustering is a purely exploratory tool. To proceed, we can either choose to (1) build separate MLR models of the $\Delta$ responses for the homes within each cluster; (2) build a single MLR model ignoring the clusters; or (3) build a single MLR model that attempts to incorporate the split in homes revealed by the clustering directly into the model. The "Heating Degree Day" (HDD) variable should suitably distinguish between the homes in each group, so for the third approach, we build a single MLR model for all homes, and we begin the model-building process not only with the original 49 variables, but also including the 48 interaction terms between HDD and each of the remaining variables. Then, we apply backward selection until all remaining variables in the model are significant. This approach did not produce a better model than the first or second types of models, so we do not report on its results. We simply mention this third approach to demonstrate that trying to sensibly split this complex dataset only on the basis of HDD could not replicate the information contained in the cluster analysis. 


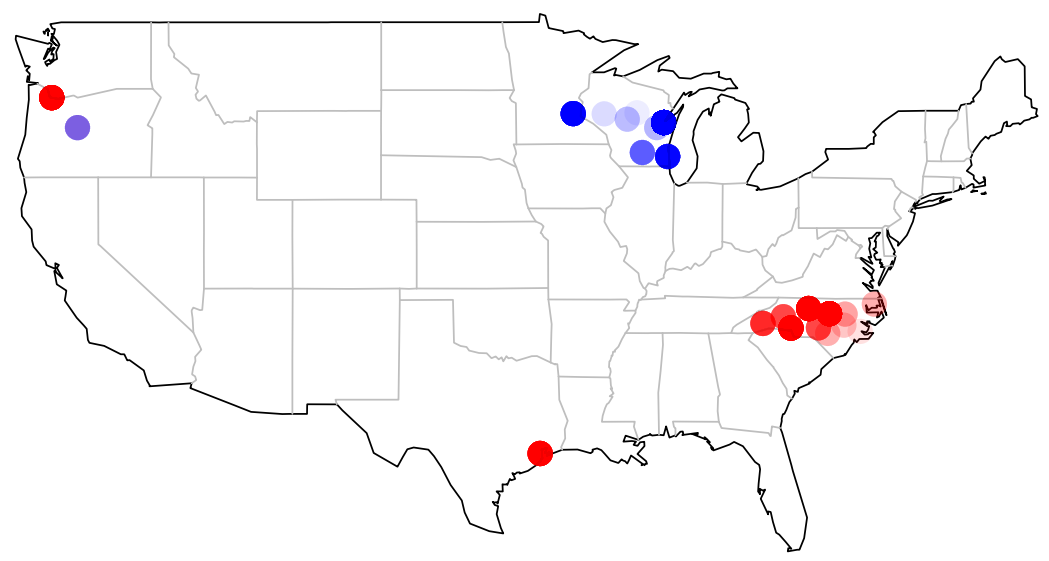

Figure 4: Plot of the location of homes at city level for both clusters. Cities with red circles indicate that the homes are located in the first Warm cluster, while cities with blue circles indicate that the homes are located in the second Cold cluster. The blue city in Oregon contains homes in both clusters.

\subsection{MLR Models}

In this section, we first describe the fit of the MLR models both overall and within each cluster and analyze the types of variables included in each model. Then, we show that the predictive ability of the separate Warm and Cold cluster models is superior to the overall model, which clearly demonstrates the benefit of two clusters over none. Finally, we show the improvements in the RBES predictions after adjusting each home's energy usage based on the MLR models.

\subsubsection{Fitted MLR Models}

Of the 997 and 709 homes used for the electricity and natural gas analyses, 121 are missing information on measured energy; 9 are not successfully simulated in BEopt; and 1 home does not have measured energy and is also 
Table 2: Table of Adjusted $R^{2}$ values for both responses and the number of homes, $n$, present in each regression.

\begin{tabular}{|l|rr|lr|}
\hline & \multicolumn{2}{|c|}{$\Delta$ Electricity } & \multicolumn{2}{|c|}{$\Delta$ Natural Gas } \\
Grouping & $n$ & Adj. $R^{2}$ & $n$ & Adj. $R^{2}$ \\
\hline \hline All Homes & 840 & $16.8 \%$ & 554 & $44.6 \%$ \\
Both Clusters & 859 & $18.4 \%$ & 556 & $44.0 \%$ \\
\hline Warm Cluster & 419 & $29.2 \%$ & 142 & $47.3 \%$ \\
Cold Cluster & 440 & $8.2 \%$ & 414 & $42.9 \%$ \\
\hline
\end{tabular}

not successfully simulated. The full sets of homes were used in the clustering analysis but not in the subsequent MLRs. Therefore, the MLRs are built using $n=866$ homes in the BAFDR dataset for the $\Delta$ electricity response and using $n=578$ homes with non-zero natural gas usage for the $\Delta$ natural gas response. Some missing explanatory variable values cause the actual number of homes used in the fit to differ from these sample sizes, as shown in Table 2. Table 2 also lists the adjusted $R^{2}$ values from each model for both responses. The Warm cluster has the largest adjusted $R^{2}$ value for both $\Delta$ electricity and $\Delta$ natural gas. A weighted average of the Warm and Cold clusters based on the number of observations in each one is used to compute an adjusted $R^{2}$ for "Both Clusters," and taken together, the clusters explain more of the variability in $\Delta$ electricity than the model built on all homes, and they explain roughly the same amount of variability in $\Delta$ natural gas as the overall model.

Tables 3 and 4 list the variables included in each of the overall, Warm cluster, and Cold cluster models along with each one's associated $p$-value for 
the $\Delta$ electricity and $\Delta$ natural gas responses, respectively. The variables are sorted into two columns, one for the variables with positive coefficients in the MLR and one for the variables with negative coefficients. Within each column, the variables are sorted with those that are most significant at the top. Variables with a positive estimated coefficient lead in the direction of overpredicting electricity consumption, while variables with a negative estimated coefficient lead in the direction of underpredicting electricity consumption. For example, Age of Home has a positive coefficient, so the older the home, the more likely it becomes that the $\Delta$ electricity will be positive (i.e., RBES overpredicts electricity) as well.

For the $\Delta$ electricity response in Table 3 , we note important groups of variables. No Garage, Conditioned Floor Area, and East and South Window Area are all geometry related variables and are processed to be used in BEopt. Since BEopt requires a 3-dimensional closed geometry, this may indicate that the RBES process could be improved in this area (e.g., improve the translation algorithms used to create the 3-D geometry from the audit data). Furthermore, Above Grade Wall U-value and Dishwasher Energy Factor (EF) are from the intermediate steps in the translation scripts, so information for these variables has potentially been lost in that process or could be processed more effectively.

Number of Bedrooms and Age of Home appear in both cluster models and in the overall model for $\Delta$ electricity. In this RBES process, miscellaneous electric loads are calculated based on the number of bedrooms and floor area of a home, so the presence of Number of Bedrooms in these models could be a proxy for occupant behavior, and its presence in these models suggests 
that miscellaneous electric loads may not be completely accounted for in the calculation. In addition, the quantitative Age of Home is not used directly in the RBES simulation process, so one possible explanation for its presence in these models is the effect of RBES defaults on older homes. Particular characteristics, like heating thermostat set points, are defaulted in BEopt because the BAFDR data were collected for asset analyses, and therefore no information on occupant behavior is known. Since the defaults are based on benchmark values given by the Building America House Simulation Protocols, it is possible that they are not representative for older homes. For example, older homes are typically less energy efficient than newer homes, which could lead to a lower heating set points than the benchmark value since they would be more expensive to heat. This possible error in the assumed heating set point would contribute to overprediction.

Similar to the $\Delta$ electricity models, there are several geometry related and duct and ventilation variables present in the $\Delta$ natural gas models listed in Table 4, like Cathedral Ceiling Area, East and South Window Area, Conditioned Floor Area, Duct Number of Returns, and Duct Location. Since translating these variables from the original data set into inputs to be used in BEopt is a particularly difficult process, some information from these variables is likely lost or distorted. In particular, Living Space ACH50 is a significant variable in all models for the $\Delta$ natural gas response, and one explanation for its presence is that there was a calculation error (that was subsequently identified and corrected) in the translation scripts. The correction was related to leakage area, which is calculated using Living Space $\mathrm{ACH} 50$. 
Table 3: List of variables that lead to increasing (left column) and decreasing (right column) $\Delta$ electricity in the model for all homes, the Warm cluster, and the Cold cluster and their $p$-values.

\begin{tabular}{|c|c|c|c|}
\hline \multicolumn{2}{|l|}{ Positive Coefficient } & \multicolumn{2}{|l|}{ Negative Coefficient } \\
\hline Variables & $p$-value & Variables & $p$-value \\
\hline \multicolumn{4}{|c|}{ Model with All Homes } \\
\hline Age of Home & $3.64 \mathrm{e}-05$ & Number of Bedrooms & $8.88 \mathrm{e}-06$ \\
\hline AH Leak RA & 0.0014 & Enclosed Crawlspace Perimeter & $5.70 \mathrm{e}-05$ \\
\hline Roof Color: Dark & 0.0025 & Duct Location: Garage & 0.0014 \\
\hline Unconditioned Duct R-value & 0.0068 & Left Window U-value & 0.0031 \\
\hline Ventilation Type: Balanced & 0.0130 & No Garage & 0.0035 \\
\hline \multirow[t]{4}{*}{ Conditioned Floor Area } & 0.0221 & East Window Area & 0.0040 \\
\hline & & House Vent Fan Power & 0.0068 \\
\hline & & Dishwasher EF & 0.0140 \\
\hline & & No Furnace & 0.0396 \\
\hline \multicolumn{4}{|c|}{ Model for Warm Cluster } \\
\hline Above Grade Wall U-value & $1.74 \mathrm{e}-05$ & Number of Bedrooms & $1.30 \mathrm{e}-06$ \\
\hline Age of Home & $2.54 \mathrm{e}-05$ & Duct Location: Garage & $8.55 \mathrm{e}-06$ \\
\hline South Window Area & 0.0001 & Left Window U-value & 0.0003 \\
\hline Unconditioned Duct R-value & 0.0012 & No Garage & 0.0005 \\
\hline Right Window U-value & 0.0034 & Water Heater Tank Volume & 0.0020 \\
\hline \multirow[t]{5}{*}{ AH Leak RA } & 0.0043 & Dishwasher EF & 0.0022 \\
\hline & & Living Space ACH50 & 0.0234 \\
\hline & & Duct Number of Returns & 0.0236 \\
\hline & & Duct Location: Unfinished Bsmt & 0.0443 \\
\hline & & No Furnace & 0.0457 \\
\hline \multicolumn{4}{|c|}{ Model for Cold Cluster } \\
\hline Age of Home & 0.0016 & East Window Area & 0.0035 \\
\hline Ventilation Type: Balanced & 0.0057 & Azimuth: 0 & 0.0054 \\
\hline Roof Color: Dark & 0.0112 & Number of Bedrooms & 0.0171 \\
\hline Conditioned Floor Area & 0.0142 & House Vent Fan Power & 0.0197 \\
\hline Water Heater Energy Factor & 0.0223 & & \\
\hline
\end{tabular}


Above Grade Wall U-value is the most significant variable in both the overall and Cold cluster models. As the Above Grade Wall U-value increases, the average $\Delta$ natural gas also increases. Above Grade Wall U-value is an intermediate variable calculated in the translation scripts and is not used directly in BEopt, so it is possible that the variables created from Above Grade Wall U-value for use in BEopt mishandle the information present in this variable. Or, for example, there could be inherent issues with the hard-wired assumptions and modeling algorithms in the simulation tool for poorly-insulated wall assemblies (walls with high U-values).

Finally, several variables appear in the model for the Warm cluster or the model for the Cold cluster that do not appear in the model with all homes for both of the $\Delta$ responses. These variables provide additional information as to where improvements may be made in the RBES process for both groups of homes when simulating electricity or natural gas consumption. For example, an "Enclosed Crawlspace Perimeter" is significant in explaining the $\Delta$ natural gas for Cold cluster homes but is not significant in the model for all homes. This could indicate the presence of translation or modeling errors for enclosed crawlspaces that are most pronounced in colder climates (e.g., crawlspace insulation assumptions or ground-coupling models). These results indicate that while similar variables appear in all models for each of the $\Delta$ responses, when the homes are broken into clusters, additional information is gained on which areas may need improvement in the simulation process for homes of these particular types. 
Table 4: List of variables that lead to increasing (left column) and decreasing (right column) $\Delta$ natural gas consumption in the models for all homes, the Warm cluster, and the Cold cluster and their $p$-values.

\begin{tabular}{|c|c|c|c|}
\hline \multicolumn{2}{|l|}{ Positive Coefficient } & \multicolumn{2}{|l|}{ Negative Coefficient } \\
\hline Variables & $p$-value & Variables & $p$-value \\
\hline \multicolumn{4}{|c|}{ Model with All Homes } \\
\hline Above Grade Wall U-value & $4.44 \mathrm{e}-13$ & East Window Area & 0.0002 \\
\hline Heating Degree Days & $1.16 \mathrm{e}-12$ & South Window Area & 0.0019 \\
\hline Cathedral Ceiling Area & $2.44 \mathrm{e}-11$ & Water Heater Energy Factor & 0.0028 \\
\hline Duct Location: Unfinished Attic & $1.23 \mathrm{e}-08$ & & \\
\hline Living Space ACH50 & $5.03 \mathrm{e}-06$ & & \\
\hline Conditioned Floor Area & 0.0006 & & \\
\hline Age of Home & 0.0033 & & \\
\hline Unfinished Attic U-value & 0.0047 & & \\
\hline Duct Number of Returns & 0.0075 & & \\
\hline AH Leak RA & 0.0164 & & \\
\hline \multicolumn{4}{|c|}{ Model for Warm Cluster } \\
\hline Living Space ACH50 & $7.67 \mathrm{e}-08$ & Azimuth: 0 & 0.0266 \\
\hline Unfinished Attic U-value & $1.09 \mathrm{e}-05$ & & \\
\hline Fixed Heating Capacity & $9.35 \mathrm{e}-05$ & & \\
\hline Cathedral Ceiling Area & 0.0009 & & \\
\hline \multicolumn{4}{|c|}{ Model for Cold Cluster } \\
\hline Above Grade Wall U-value & $9.54 \mathrm{e}-11$ & East Window Area & 0.0001 \\
\hline Cathedral Ceiling Area & $5.21 \mathrm{e}-09$ & South Window Area & 0.0012 \\
\hline Living Space ACH50 & $6.04 \mathrm{e}-07$ & & \\
\hline Duct Location: Unfinished Attic & $3.06 \mathrm{e}-05$ & & \\
\hline Conditioned Floor Area & 0.0008 & & \\
\hline Duct Number of Returns & 0.0015 & & \\
\hline Heating Degree Days & 0.0069 & & \\
\hline Use Ceiling Fan & 0.0180 & & \\
\hline Dishwasher EF & 0.0219 & & \\
\hline Left Window Percent of Total & 0.0228 & & \\
\hline Enclosed Crawlspace Perimeter & 0.0473 & & \\
\hline
\end{tabular}


Table 5: $C V_{M S E}$ for all responses with the largest $5 \%$ of squared errors removed.

\begin{tabular}{|l|cc|}
\hline Grouping & $\Delta$ Electricity & $\Delta$ Natural Gas \\
\hline \hline All Homes & 99.64 & 470.63 \\
Both Clusters & $\mathbf{9 7 . 5 9}$ & $\mathbf{4 0 8 . 6 8}$ \\
\hline Warm Cluster & 102.85 & $\mathbf{3 2 9 . 7 9}$ \\
Cold Cluster & $\mathbf{9 2 . 6 9}$ & 433.47 \\
\hline
\end{tabular}

\subsubsection{Leave-One-Out Cross-Validation}

To demonstrate the utility of constructing models within each cluster as opposed to one overall model, we compare the predictive capabilities of these models using leave-one-out $\mathrm{CV}$. Table 5 gives the $C V_{M S E}$ computed using the algorithm described in Section 3.2.1. A few observations that have unusually high squared errors are observations that already have unusually high or low response values. Therefore, the largest $5 \%$ of $\mathrm{CV}$ squared errors are removed. In Table 5, the $C V_{M S E}$ values for all homes, both clusters, the Warm cluster, and the Cold cluster with the largest $5 \%$ of the squared errors removed are given, where "Both Clusters" denotes the weighted average of the Warm and Cold clusters. For both responses, the weighted average $C V_{M S E}$ of the Warm and Cold clusters is smaller than the $C V_{M S E}$ for all homes. Therefore, splitting the homes into two clusters produces models with better predictive capabilities than does a single model using all homes. We also see that $\Delta$ electricity is more predictable for Cold cluster homes, and $\Delta$ natural gas is more predictable for Warm cluster homes. This may be due to the fact that electricity is more likely to be used for cooling, so there is less electricity consumption in Cold cluster homes (and vice versa for natural gas). 


\subsubsection{Model Output Adjustments}

Using the cross-validation predictions, we can now adjust the RBES predicted energy consumption of a home by its predicted $\Delta$ (the fitted value of the model) and use the adjusted predictions to calculate the adjusted $\Delta$ for electricity and natural gas consumption, as shown in Equation (4). Table 6 shows the mean and standard deviation of the adjusted $\Delta$ electricity and adjusted $\Delta$ natural gas responses for all models. Compared with the summaries of the original $\Delta$ responses in Table 1 , the adjusted $\Delta$ responses are now centered near zero with smaller standard deviations. The $\Delta$ electricity standard deviations have been reduced for the All Home, Warm Cluster, and Cold Cluster models by $7 \%, 13 \%$, and $3 \%$, respectively. The variability in the natural gas errors have been reduced more substantially with reductions in standard deviations for the All Home, Warm Cluster, and Cold Cluster models of $27 \%, 24 \%$, and $26 \%$, respectively.

Visually, the bottom panels of Figure 1 show the adjusted predicted energy consumption based on the cluster models plotted against the measured energy consumption for both electricity and natural gas. The most striking results are that (1) the substantial bias in the electricity predictions has been corrected, since the points align with the line of perfect agreement; and (2) the variability in the natural gas predictions has been significantly reduced, as shown by the reduced scatter around the line of perfect agreement.

\section{Conclusions}

The goal of this work was to efficiently use available information on a set of homes to build statistical models that would be used to improve the accuracy 
Table 6: Mean and standard deviation of the $\Delta$ responses when predictions are adjusted by the fitted value of the MLR models.

\begin{tabular}{|l|rr|rr|}
\hline & \multicolumn{2}{|c|}{ Adj. $\Delta$} & \multicolumn{2}{c|}{ Adj. $\Delta$} \\
Model & \multicolumn{2}{|c|}{ Electricity } & \multicolumn{2}{c|}{ Natural Gas } \\
\hline \hline All Homes & 0.006 & 11.86 & -0.028 & 23.48 \\
Both Clusters & 0.014 & 11.51 & -0.020 & 23.76 \\
\hline Warm Cluster & 0.016 & 11.75 & -0.028 & 23.28 \\
Cold Cluster & 0.011 & 11.30 & -0.017 & 23.95 \\
\hline
\end{tabular}

of annual energy consumption predictions of an RBES simulation tool. To achieve this goal, we (1) removed redundant information in the variable set; (2) clustered the homes into two groups; and (3) created MLR models both for all homes and within each cluster. Creating models based on these two clusters produced better predictions of the error in annual electricity and natural gas use than building a single model based on all homes, as shown by the higher adjusted $R^{2}$ values and lower cross-validation MSE's. However, the adjusted predictions are very similar regardless of whether the clusters are used or not. These models also point to areas of potential improvement both in the RBES tool and in the translation algorithms. Differences in variables selected for the two clusters provide insight on factors that could be improved in the simulation process for homes in those groups. In this example, the MLR model even identified a variable that was incorrectly coded in the translation script.

Ultimately, the fitted MLR models are used to adjust the RBES predic- 
tions independently for each home, and the reductions in bias and variability are substantial. Even without grouping the homes, the adjusted predictions are much improved, so the approach described here can be applied to postprocess the predictions of any RBES tool. When removing one home at a time to assign it to a cluster, fit the MLR model, and adjust its prediction, we are assuming that the removed home is similar to the homes in the BAFDR database. The BAFDR database that is used in this work is not representative of all detached single-family homes in the United States, so the clusters and models should be updated as new homes are added to the BAFDR. However, ideally, when a new home is simulated in an RBES program, it would be placed in the group to which its characteristics are the most similar, and the model for that group would be used to adjust the home's RBES annual energy consumption prediction.

We have described a method for post-processing RBES predictions to improve accuracy, but we also recognize the value of the MLR analysis in identifying potential improvements to the RBES process. As a general approach, significant variables identified in the MLR should be investigated and an effort should be made to correct errors in the translation process and/or simulation tool before developing the "final" MLR post-processing models. Fixing these errors should generally reduce the magnitude of the corrections made from post-processing and should also improve the predictive capability of the RBES tool when analyzing scenarios such as energy saving upgrades. In this study, we used a developmental version of the translation scripts and only created the post-processing models to demonstrate how they could be applied to improve predictions; we plan to use the MLR results to improve 
the RBES process and then generate updated post-processing models using a larger set of homes from the BAFDR. Potential future work related to this study includes: (a) finding ways to post-process RBES predictions of energy savings for retrofit measures; (b) automating the variable selection in the MLR models with the LASSO method [30]; and (c) automating the removal of collinear variables with a decision tree algorithm [31]. Automation of the methods applied in this study would allow them to be included in industry software testing procedures, which would increase the amount of actionable information test takers have to improve the accuracy of their RBES tools and associated predictions.

\section{Acknowledgements}

This work was supported by the U.S. Department of Energy under Contract No. DE-AC36-08GO28308 with the National Renewable Energy Laboratory. The project was completed under the Alliance Partner University Program. The authors would like to thank David Roberts for support and feedback on this project and Joseph Robertson for his assistance in preparing the BEopt and BAFDR datasets for analysis. Finally, the authors would like to thank those who have contributed data to the Building America Field Data Repository and to the anonymous referees whose comments have improved the content and presentation of this work.

\section{References}

[1] Annual energy review 2010, 2011. URL: http://www.eia.gov/ totalenergy/data/annual/pdf/aer.pdf. 
[2] B. Polly, N. Kruis, D. Roberts, Assessing and Improving the Accuracy of Energy Analysis for Residential Buildings, Technical Report, National Renewable Energy Laboratory, 2011.

[3] K. Lomas, H. Eppel, C. Martin, D. Bloomfield, Empirical validation of building energy simulation programs, Energy and Buildings 26 (1997) 253-275.

[4] L. Tronchin, K. Fabbri, Energy performance building evaluation in Mediterranean countries: Comparison between software simulations and operating rating simulation, Energy and Buildings 40 (2008) 1176-1187.

[5] R. Brown, Modeled vs. Actual Energy Savings for Energy Upgrade California Home Retrofits, Technical Report PIR-08-018, BKi, Oakland, CA, 2012.

[6] D. Roberts, N. Merket, B. Polly, M. Heaney, S. Casey, J. Robertson, Assessment of the U.S. Department of Energy's Home Energy Scoring Tool, Technical Report TP-5500-54074, National Renewable Energy Laboratory, Golden, CO, 2012.

[7] SEEM 94 Calibration to Single Family RBSA Data, Technical Report, Regional Technical Forum, Portland, OR, 2013. URL: http://rtf. nwcouncil .org/subcommittees/seem/Meetings/meetings.htm.

[8] H. R. Glahn, D. A. Lowry, The use of model output statistics (MOS) in objective weather forecasting, Journal of Applied Meteorology 11 (1972) 1203-1211. 
[9] W. H. Klein, H. R. Glahn, Forecasting local weather by means of model output statistics, Bulletin of the American Meteorological Society 55 (1974) 1217-1227.

[10] E. Eccel, L. Ghielmi, P. Granitto, R. Barbiero, F. Grazzini, D. Cesari, Prediction of minimum temperatures in an Alpine region by linear and non-linear post-processing of meteorological models, Nonlinear Processes in Geophysics 14 (2007) 211-222.

[11] T. Gneiting, A. E. Raftery, A. H. Westveld, T. Goldman, Calibrated probabilistic forecasting using ensemble model output statistics and minimum CRPS estimation, Monthly Weather Review 133 (2004) 10981118.

[12] J. Chenevez, C. O. Jensen, Operational ozone forecasts for the region of Copenhagen by the Danish Meteorological Institute, Atmospheric Environment 35 (2001) 4567-4580.

[13] J. M. Sloughter, T. Gneiting, A. E. Raftery, Probabilistic wind speed forecasting using ensembles and Bayesian model averaging, Journal of the American Statistical Association 105 (2010) 25-35.

[14] H. Paeth, N. M. J. Hall, M. A. Gaertner, M. D. Alonso, S. Moumouni, J. Polcher, P. M. Ruti, A. H. Fink, T. L. M. Gosset, A. T. Gaye, D. P. Rowell, W. Moufouma-Okia, D. Jacob, B. Rockel, F. Giorgi, M. Rummukainen, Progress in regional downscaling of West African precipitation, Atmospheric Science Letters 12 (2011) 75-82. 
[15] M. Santamouris, G. Mihalakakou, P. Patargias, N. Gaitani, K. Sfakianaki, M. Papaglastra, C. Pavlou, P. Doukas, E. Primikiri, V. Geros, M. Assimakopoulos, R. Mitoula, S. Zerefos, Using intelligent clustering techniques to classify the energy performance of school buildings, Energy and Buildings 39 (2007) 45-51.

[16] S. Petcharat, S. Chungpaibulpatana, P. Rakkwamsuk, Assessment of potential energy saving using cluster analysis: A case study of lighting systems in buildings, Energy and Buildings 52 (2012).

[17] K. Kissock, J. Haberl, D. Ciaridge, Development of a toolkit for calculating linear, change-point linear and multiple-linear inverse building energy analysis models, Technical Report 1050-RP, ASHRAE, 2002. URL: http://repository.tamu .edu/bitstream/handle/1969. 1/2847/ESL-TR-02-11-02.pdf? sequence=1.

[18] R. Hendron, C. Engebrecht, Building America House Simulation Protocols, Technical Report TP-550-49426, National Renewable Energy Laboratory, Golden, CO, 2010.

[19] S. Sheather, A Modern Approach to Regression with R, Springer Science+Business, New York, 2010.

[20] J. Guilford, Psychometric Methods, McGrawHill Book Company, Inc, New York, NY, 1942.

[21] G. Glass, K. Hopkins, Statistical Methods in Education and Psychology, 2nd ed., Prentice-Hall, Englewood Cliffs, NJ, 1984. 
[22] R. Johnson, D. Wichern, Applied Multivariate Statistical Analysis, 6th ed., Pearson Education, Upper Saddle River, NJ, 2007.

[23] J. Ward, Hierarchical grouping to optimize an objective function, Journal of the American Statistical Association 48 (1963) 236-244.

[24] A. Izenman, Modern Multivariate Statistical techniques, Springer Science+Business, New York, NY, 2008.

[25] X. L. Xie, G. Beni, Validity measure for fuzzy clustering, IEEE Transactions on Pattern Analysis and Machine Intelligence 3 (1991) 841-847.

[26] L. Hubert, J. Schultz, Quadratic assignments as a general data-analysis strategy, British Journal of Mathematical and Statistical Psychology 29 (1976) 190-241.

[27] J. Dunn, Well separated clusters and optimal fuzzy partitions, Journal of Cybernetics 4 (1974) 95-104.

[28] J. Neter, M. Kutner, W. Wasserman, C. Nachtsheim, Applied Linear Statistical Models, 4th ed., McGraw-Hill, Boston, MA, 1996.

[29] D. Wilks, Statistical Methods in the Atmospheric Sciences, 3rd ed., Elsevier Inc., Oxford, UK, 2011.

[30] R. Tibshirani, Regression shrinkage and selection via the lasso, Journal of the Royal Statistical Society, Series B 58 (1996) 267-288.

[31] L. Breiman, J. Friedman, R. Olshen, C. Stone, Classification and Regression Trees, Wadsworth, Boca Raton, FL, 1984. 